Pacific Journal of Mathematics

RELATION OF A DIRECT LIMIT GROUP TO ASSOCIATED
VECTOR GROUPS 


\title{
RELATION OF A DIRECT LIMIT GROUP TO \\ ASSOCIATED VECTOR GROUPS
}

\author{
PaUl D. Hill
}

A set $M$ with a binary, transitive relation $<$ is said to be directed if for each pair $a, b$ in $M$, there is a $c$ in $M$ such that $a<c, b<c$. Let $\left\{G_{a}\right\}_{a \in M}$ be a collection of groups indexed by a directed set $M=\{a, b, \cdots$; $<\}$, and for each $a<b$ in $M$ let $h_{b}^{a}$ be a homomorphism of $G_{a}$ into $G_{b}$. The homomorphisms are assumed to satisfy the relations

and

(i) $h_{c}^{b} h_{b}^{a}=h_{c}^{a}$ if $a<b<c$

(ii) if $a<a$, then $h_{a}^{a}$ is the identity.

We call such a system a direct system of groups and define a direct limit group of this system in the following manner. Two elements $g_{a} \in G_{a}$ and $\bar{g}_{b} \in g_{b}$ are said to be equivalent if there is a $c>a, b$ such that $h_{c}^{a}\left(g_{a}\right)=h_{c}^{b}\left(\bar{g}_{b}\right)$. Let $g_{a}^{*}$ denote the collection of elements which are equivalent to $g_{a}$. Now given any two equivalence classes $g_{a}^{*}$ and $\bar{g}_{b}^{*}$, there exists a $c$ and elements $g_{c}, \bar{g}_{c}$ in $G_{c}$ such that $g_{a}^{*}=g_{c}^{*}$ and $\bar{g}_{b}^{*}=\bar{g}_{c}^{*}$. We define $g_{a}^{*} \cdot \bar{g}_{b}^{*}=\left(g_{c} \bar{g}_{c}\right)^{*}$. This multiplication is a well defined binary operation on the set, $G^{*}$, of equivalence classes. And it may be shown that $G^{*}$ is a (multiplicative) group, which we define to be the direct limit group of the given system.

Let $G=\Pi G_{a}$ be the restricted direct product of the given groups $G_{a}$, and consider the groups $G_{a}$ as subgroups of $G$. An element in $G$ of the form $g_{a}^{-1} h_{b}^{a}\left(g_{a}\right)$ is called a relation. Let $H$ be the subgroup generated by the relations of $G$. Note that the inverse of a relation is a relation. By a "last" element of $M$ we mean an element $b$ such that $a<b$ for all $a$ in $M$. If $M$ contains no last element, it is immediate that given $a_{1}, a_{2}, \cdots, a_{k}$ in $M$, there exists a $b \in M$ with the property $a_{i}<b, a_{i} \neq$ $b$ for $i=1,2, \cdots, k$.

LEMMA 1. If $M$ contains no last element, the commutator group $K$ of $G$ is contained in $H$.

Proof. Let $x=g_{a_{1}} g_{a_{2}} \cdots g_{a_{k}}$ and $y=\bar{g}_{b_{1}} \bar{g}_{b_{2}} \cdots \bar{g}_{b}$, be arbitrary elements of $G$, where $a_{m}=a_{n}$ or $b_{m}=b_{n}$ implies that $m=n$. First choose $a$ with the property that $a_{i}<a, a_{i} \neq a$, and $b_{i} \neq a$ for all $i$. Then choose $b$ such that $b_{i}<b, b_{i} \neq b, a_{i} \neq b$, and $a \neq b$. We have

$$
x y x^{-1} y^{-1}=\prod_{i=1}^{k} g_{a_{i}} \prod_{i=1}^{j} \bar{g}_{b_{i}} \prod_{i=k}^{1} g_{a_{i}}^{-1} \prod_{i=j}^{1} \bar{g}_{b_{i}}^{-1}
$$

Received September 8, 1959, and in revised form September 11, 1959. 


$$
\begin{aligned}
& =\prod_{i=1}^{k} g_{a_{i}} \prod_{i=1}^{k} h_{a}^{a_{i}}\left(g_{a_{i}}^{-1}\right) \prod_{i=1}^{j} \bar{g}_{b_{i}} \prod_{i=1}^{j} h_{b}^{b_{i}}\left(\bar{g}_{b_{i}}^{-1}\right) \\
& =\prod_{i=k}^{1} g_{a_{i}}^{-1} \prod_{i=k}^{1} h_{a}^{a_{i}}\left(g_{a_{i}}\right) \prod_{i=j}^{1} \bar{g}_{b_{i}}^{-1} \prod_{i=j}^{1} h_{b}^{b_{i}}\left(\bar{g}_{b_{i}}\right) \\
& =\prod_{i=1}^{k} g_{a_{i}} h_{a}^{a_{i}}\left(g_{a_{i}}^{-1}\right) \prod_{i=1}^{i} \bar{g}_{b_{i}} h_{b}^{b_{i}}\left(\bar{g}_{b_{i}}^{-1}\right) \prod_{i=k}^{1} g_{a_{i}}^{-1} h_{a}^{a_{i}}\left(g_{a_{i}}\right) \prod_{i=j}^{1} \bar{g}_{b_{i}}^{-1}\left(\bar{g}_{b_{i}}\right) .
\end{aligned}
$$

Thus $x y x^{-1} y^{-1} \in H$. Since $H$ is a group, the lemma follows.

CoRollary 1. If $M$ contains no last element, then $H$ is a normal subgroup of $G$.

The following example shows that $H$ may not be normal in $G$ if $M$ contains a last element.

ExAmPLe. Suppose that $M$ is $\left\{1,2\right.$; $\leqq$. Let $G_{2}$ be the symmetric group on the set $\{1,2,3\}$, and let $G_{1}$ be the subgroup of $G_{2}$ of those elements fixing 3. Define $h_{2}^{1}$ to be the identity isomorphism of $G_{1}$ into $G_{2}$. Then $H$, a cyclic group of order 2 , is generated by $((1,2),(1,2))$. It is, therefore, not normal in $G$.

Lemma 2. If $g_{a}$ in $G_{a}$ is in $H$, then there exists $a b$ such that $h_{b}^{a}\left(g_{a}\right) \in K_{b}$, the commutator group of $G_{b}$.

Proof. In general, if $x_{a}$ in $G_{a}$ is the product $x_{a_{1}} x_{a_{2}} \cdots x_{a_{n}}$ where $x_{a_{i}} \in G_{a_{i}}$ and if $b>a, a_{i}$ for $i=1,2, \cdots, n$, then $h_{b}^{a}\left(x_{a}\right)$ can be written as the product of the elements $h_{b}^{a}\left(x_{a_{1}}\right), h_{b}^{a_{2}}\left(x_{a_{2}}\right), \cdots, h_{b}^{a_{n}}\left(x_{a_{n}}\right)$ in some order. This fact is easily proved by induction on $n$. If $n>1$, by the induction hypothesis we may as well assume that the factors $x_{a_{i}}$ are nontrivial. Thus two of the factors must be contained in a single group $G_{a_{i}}$. And the product $x_{a_{1}} x_{a_{2}} \cdots x_{a_{n}}$ can be contracted to a product of the same form with one less factor by taking one of the new factors to be the product of two of the old and letting the other factors remain unchanged (except, possibly, for the order in which they appear).

Since $g_{a}$ is in $H$, it can be written in the form $\prod_{i=1}^{k} g_{a_{i}}^{-1} h_{b_{i}}^{a_{i}}\left(g_{a_{i}}\right)$. Choose $b$ such that $b>a, b_{i}$ for $i=1,2, \cdots, k$. Then

$$
\begin{aligned}
h_{b}^{a}\left(g_{a}\right) K_{b} & =\prod_{i=1}^{k} h_{b}^{a_{i}}\left(g_{a_{i}}^{-1}\right) h_{b}^{b i} h_{b_{i}}^{a_{i}}\left(g_{a_{i}}\right) K_{b} \\
& =\prod_{i=1}^{k} h_{b}^{a_{i}}\left(g_{a_{i}}^{-1}\right) h_{b}^{a_{i}}\left(g_{a_{i}}\right) K_{b}=1_{b} K_{b}=K_{b},
\end{aligned}
$$

which proves the lemma.

THEOREM 1. If $H$ is a normal subgroup of $G$, then $G / H$ is a homomorphism image of $G^{*}$, where the kernel of the homomorphism is contained in the commutator subgroup, $K^{*}$ 
REMARKs. The theorem is well known [1] in case the groups $G_{a}$ are abelian. In this case $H$ is necessarily normal and $K^{*}=1$ is the identity. Thus $G^{*} \cong G / H$, and we have two equivalent definitions for the direct limit.

Proof of theorem. Let $f$ be the mapping of $G^{*}$ into $G / H$, defined by: $g_{a}^{*} \rightarrow g_{a} H$. In order to show that $f$ is single-valued, let $g_{a}^{*}=\bar{g}_{b}^{*}$. There exists $c>a, b$ such that $h_{c}^{a}\left(g_{a}\right)=h_{c}^{b}\left(\bar{g}_{b}\right)$. Thus $g_{a}^{-1} \bar{g}_{b}=g_{a}^{-1} h_{c}^{a}\left(g_{a}\right) h_{c}^{b}\left(\bar{g}_{b}\right)^{-1} \bar{g}_{b}$. Since $h_{c}^{b}\left(\bar{g}_{b}\right)^{-1} \bar{g}_{b}=\bar{g}_{b} h_{c}^{b}\left(\bar{g}_{b}^{-1}\right)$, we have $g_{a}^{-1} \bar{g}_{b} \in H$, which implies that $f$ is independent of the representative of $g_{a}^{*}$. The multiplicative property of $f$ is immediate. We next show that $f$ is onto. Let $g H \in G / H$ and let $g=g_{a_{1}} g_{a_{2}} \cdots g_{a_{k}}$, where the $a_{i}$ 's are distinct. Choose $b$ such that $a_{i}<b$ for $i=1,2, \cdots, k$. If $a_{i}=b$ for some $i$, we may as well assume that $i=k$ since the $g_{a_{i}}$ 's commute. For each $i, g_{a_{i}}^{-1} h_{b}^{a_{i}}\left(g_{a_{i}}\right) \in H$. Thus

$$
\bar{g}=\prod_{i=k}^{1} g_{a_{i}}^{-1} h_{b}^{a_{i}}\left(g_{a_{i}}\right)=\prod_{i=k}^{1} g_{a_{i}}^{-1} \prod_{i=k}^{1} h_{b}^{a_{i}}\left(g_{a_{i}}\right)
$$

is in $H$, which implies that $g \bar{g} H=g H$. But $g \bar{g}=\prod_{i=k}^{1} h_{b}^{a_{i}}\left(g_{a_{i}}\right)$ is in $G_{b}$. Hence $f\left((g \bar{g})^{*}\right)=g H$, and $f$ is onto. Since $g_{a} \in K_{a}$, the commutator group of $G_{a}$, implies that $g_{a}^{*} \in K^{*}$, it follows from Lemma 2 that the kernel of $f$ is contained in $K^{*}$.

THEOREM 2. If $M$ contains no last element, then $G^{*} / K^{*} \cong G / H$.

Proof. By Corollary 1, $H$ is normal in $G$. Thus by Theorem 1, we need only show that the kernel of $f$ is the whole commutator group, $K^{*}$. However, if $g_{a}^{*}$ is a commutator of $G^{*}$, then there exist a $b$ and a commutator $\bar{g}_{b} \in K_{b}$ such that $g_{a}^{*}=\bar{g}_{b}^{*}$. Since $K_{b} \subseteq K$, by Lemma 1 $\bar{g}_{b} \in H$. Thus $f\left(g_{a}^{*}\right)=H$, and the theorem follows.

The limit group $G^{*}$ is abelian if and only if for every $a$ in $M$ and for every commutator $g_{a}$ of the group $G_{a}$, there exists a $b>a$ (depending on $\left.g_{a}\right)$ such that $h_{b}^{a}\left(g_{a}\right)=1_{b}$. Also, under this condition the commutator subgroups, $K_{a}$, of the groups $G_{a}$ are contained in $H$, and $H$ is normal in $G$ since the conjugate of a generator of $H$ transformed by a general element of $G$

$$
\begin{aligned}
& \left\{x_{c}\right\}_{c \in M} g_{a}^{-1} h_{b}^{a}\left(g_{a}\right)\left\{x_{c}\right\}_{c \in M}^{-1}=x_{a} x_{b} g_{a}^{-1} h_{b}^{a}\left(g_{a}\right) x_{a}^{-1} x_{b}^{-1} \\
& \quad=x_{a} g_{a}^{-1} x_{a}^{-1} g_{a} \cdot g_{a}^{-1} h_{b}^{a}\left(g_{a}\right) \cdot h_{b}^{a}\left(g_{a}\right)^{-1} x_{b} h_{b}^{a}\left(g_{a}\right) x_{b}^{-1}
\end{aligned}
$$

remains in $H$.

Corollary 2. If the limit group $G^{*}$ is abelian, then $G^{*} \cong G / H$. Moreover, the converse holds if $M$ contains no last element. 
A directed set $M=\{a, b, \cdots ;<\}$ is said to be completely directed if for every $a$ in $M$ all but a finite number of $b$ 's in $M$ satisfy the relation $a<b$. In particular, the positive integers are completely directed by $<$.

Letting $G^{\prime}=\Pi^{\prime} G_{a}$ be the complete direct product of the given groups $G_{a}$, we have

LEMMA 3. If $M$ is completely directed and has no last element, then $G^{*}$ is contained (in the sense of isomorphism) in the factor group $G^{\prime} / G$.

Proof. Define a mapping $h$ of $G^{*}$ into $G^{\prime} / G$ by: $g_{a}^{*} \rightarrow\left\{x_{b}\right\}_{b \in M} G$, where $x_{a}=g_{a}$ and $a<b$ implies that $x_{b}=h_{b}^{a}\left(g_{a}\right)$. The coordinate $x_{b}$ may be chosen as an arbitrary element of $G_{b}$ if $b$ fails to satisfy $a \leqq b$. It may be shown that $h$ is a homomorphism with trivial kernel, which proves the lemma.

Letting $F$ be the inverse image of $h\left(G^{*}\right)$ under the natural homomorphism of $G^{\prime}$ onto $G^{\prime} / G$, we observe

COROLlaRY 3. Let $M$ satisfy the conditions of Lemma 3, and let $G^{*}$ be abelian. Then in the chain

$$
G^{\prime} \supseteqq F \supseteqq G \supseteqq H \supseteqq 1
$$

we have $F / G \cong G / H \cong G^{*}$.

\section{REFERENCE}

1. S. Lefschetz, Algebraic Topology, New York, American Mathematical Society Colloquium Publications, 1942.

AUBURN UNIVERSITY 


\section{PACIFIC JOURNAL OF MATHEMATICS}

\section{EDITORS}

David GILbarg

Stanford University

Stanford, California

\section{F. H. BRowneLL}

University of Washington

Seattle 5, Washington

\section{A. L. Whiteman}

University of Southern California Los Angeles 7, California

\section{J. PAIGe}

University of California

Los Angeles 24, California

\section{ASSOCIATE EDITORS}
E. F. BECKENBACH
T. M. CHERRY
D. DERRY

\author{
E. HEWITT \\ A. HORN \\ L. NACHBIN
}

\author{
M. OHTSUKA \\ H. L. ROYDEN \\ M. M. SCHIFFER
}

E. SPANIER

E. G. STRAUS

F. WOLF

\section{SUPPORTING INSTITUTIONS}

\author{
UNIVERSITY OF BRITISH COLUMBIA \\ CALIFORNIA INSTITUTE OF TECHNOLOGY \\ UNIVERSITY OF CALIFORNIA \\ MONTANA STATE UNIVERSITY \\ UNIVERSITY OF NEVADA \\ NEW MEXICO STATE UNIVERSITY \\ OREGON STATE COLLEGE \\ UNIVERSITY OF OREGON \\ OSAKA UNIVERSITY \\ UNIVERSITY OF SOUTHERN CALIFORNIA
}

\author{
STANFORD UNIVERSITY \\ UNIVERSITY OF TOKYO \\ UNIVERSITY OF UTAH \\ WASHINGTON STATE COLLEGE \\ UNIVERSITY OF WASHINGTON \\ AMERICAN MATHEMATICAL SOCIETY \\ CALIFORNIA RESEARCH CORPORATION \\ HUGHES AIRCRAFT COMPANY \\ SPACE TECHNOLOGY LABORATORIES \\ NAVAL ORDNANCE TEST STATION
}

\footnotetext{
Mathematical papers intended for publication in the Pacific Journal of Mathematics should be typewritten (double spaced), and the author should keep a complete copy. Manuscripts may be sent to any one of the four editors. All other communications to the editors should be addressed to the managing editor, L. J. Paige at the University of California, Los Angeles 24, California.

50 reprints per author of each article are furnished free of charge; additional copies may be obtained at cost in multiples of 50 .
}

The Pacific Journal of Mathematics is published quarterly, in March, June, September, and December. The price per volume (4 numbers) is $\$ 12.00$; single issues, $\$ 3.50$. Back numbers are available. Special price to individual faculty members of supporting institutions and to individual members of the American Mathematical Society: $\$ 4.00$ per volume; single issues, $\$ 1.25$.

Subscriptions, orders for back numbers, and changes of address should be sent to Pacific Journal of Mathematics, 2120 Oxford Street, Berkeley 4, California.

Printed at Kokusai Bunken Insatsusha (International Academic Printing Co., Ltd.), No. 6, 2-chome, Fujimi-cho, Chiyoda-ku, Tokyo, Japan.

PUBLISHED BY PACIFIC JOURNAL OF MATHEMATICS, A NON-PROFIT CORPORATION

The Supporting Institutions listed above contribute to the cost of publication of this Journal, but they are not owners or publishers and have no responsibility for its content or policies. 


\section{Pacific Journal of Mathematics}

\section{Vol. 10, No. $4 \quad$ December, 1960}

M. Altman, An optimum cubically convergent iterative method of inverting a linear bounded operator in Hilbert space . . . . . . . . . . . . . . . . . . . . . . . . . . 1107

Nesmith Cornett Ankeny, Criterion for rth power residuacity ................. 1115

Julius Rubin Blum and David Lee Hanson, On invariant probability measures I . . . . . 1125

Frank Featherstone Bonsall, Positive operators compact in an auxiliary topology ..... 1131

Billy Joe Boyer, Summability of derived conjugate series . . . . . . . . . . . . . . . . 1139

Delmar L. Boyer, A note on a problem of Fuchs . . . . . . . . . . . . . . . . . 1147

Hans-Joachim Bremermann, The envelopes of holomorphy of tube domains in infinite

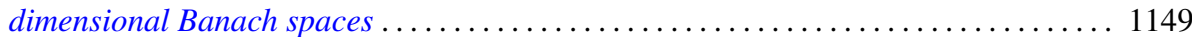

Andrew Michael Bruckner, Minimal superadditive extensions of superadditive

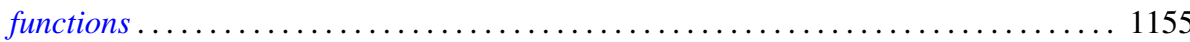

Billy Finney Bryant, On expansive homeomorphisms .................... 1163

Jean W. Butler, On complete and independent sets of operations in finite algebras . . . . . 1169

Lucien Le Cam, An approximation theorem for the Poisson binomial distribution ...... 1181

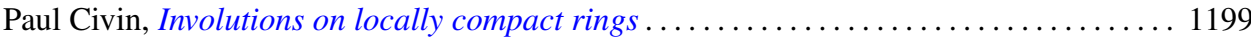

Earl A. Coddington, Normal extensions of formally normal operators . . . . . . . . . 1203

Jacob Feldman, Some classes of equivalent Gaussian processes on an interval ........ 1211

Shaul Foguel, Weak and strong convergence for Markov processes . . . . . . . . . . . 1221

Martin Fox, Some zero sum two-person games with moves in the unit interval ........ 1235

Robert Pertsch Gilbert, Singularities of three-dimensional harmonic functions . . . . . . . 1243

Branko Grünbaum, Partitions of mass-distributions and of convex bodies by

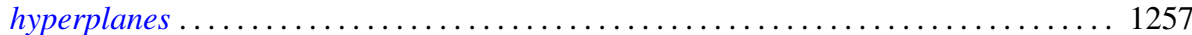

Sidney Morris Harmon, Regular covering surfaces of Riemann surfaces ........... 1263

Edwin Hewitt and Herbert S. Zuckerman, The multiplicative semigroup of integers

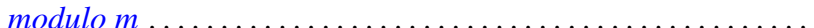

Paul Daniel Hill, Relation of a direct limit group to associated vector groups . ......... 1309

Calvin Virgil Holmes, Commutator groups of monomial groups . .

James Fredrik Jakobsen and W. R. Utz, The non-existence of expansive homeomorphisms

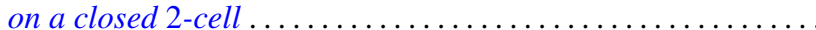

John William Jewett, Multiplication on classes of pseudo-analytic functions . . . . . . . 1323

Helmut Klingen, Analytic automorphisms of bounded symmetric complex domains . . . . 1327

Robert Jacob Koch, Ordered semigroups in partially ordered semigroups . . . . . . . . 1333

Marvin David Marcus and N. A. Khan, On a commutator result of Taussky and

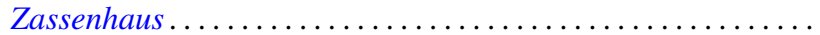

John Glen Marica and Steve Jerome Bryant, Unary algebras......

Edward Peter Merkes and W. T. Scott, On univalence of a continued fraction . . . . . . . 1361

Shu-Teh Chen Moy, Asymptotic properties of derivatives of stationary measures . . . . . 1371

John William Neuberger, Concerning boundary value problems . . . . . . . . . . . 1385

Edward C. Posner, Integral closure of differential rings . . . . . . . . . . . . . . . . . 1393

Marian Reichaw-Reichbach, Some theorems on mappings onto . . . . . . . . . . . . . 1397

Marvin Rosenblum and Harold Widom, Two extremal problems . . . . . . . . . . . . . . . . 1409

Morton Lincoln Slater and Herbert S. Wilf, A class of linear differential-difference

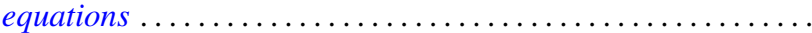

Charles Robson Storey, Jr., The structure of threads . . . . . . . . . . . . . . . . . . 1429

J. François Treves, An estimate for differential polynomials in $\partial / \partial z_{1},, \cdots, \partial / \partial z_{-} n \ldots \ldots 1447$

J. D. Weston, On the representation of operators by convolutions integrals . . . . . . . . 1453

James Victor Whittaker, Normal subgroups of some homeomorphism groups ......... 1469 